\title{
Fluoroquinolone resistance and mutational profile of gyrA gene in pulmonary MDR tuberculosis patients
}

\section{Saba Kabir}

University of the Punjab, Lahore, Pakistan

\section{Zarfishan Tahir}

Institute of Public Health, Lahore, Pakistan

\section{Nadia Mukhtar}

University of Veterinary and Animal Sciences, Lahore

\section{Muhammad Sohail}

University of the Punjab, Lahore, Pakistan

Muhammad Saqalein

Government College University Faisalabad

Abdul Rehman ( $\sim$ rehman_mmg@yahoo.com )

University of the Punjab

\section{Research article}

Keywords: XDR-Tuberculosis; Mycobacterium tuberculosis; flouroquinolone resistance; MTBDRsl assay.

Posted Date: October 7th, 2019

DOI: https://doi.org/10.21203/rs.2.15811/v1

License: (1) This work is licensed under a Creative Commons Attribution 4.0 International License. Read Full License

Version of Record: A version of this preprint was published at BMC Pulmonary Medicine on May 11th, 2020. See the published version at https://doi.org/10.1186/s12890-020-1172-4. 


\section{Abstract}

Background In recent year's high proportion of flouroquinolone (FQs) resistance in Mycobacterium tuberculosis isolates has been observed. The multidrug resistant TB is a serious risk for its transition to XDR-TB. Methods A total of 562 samples were included in the study from patients with pulmonary TB which had been on anti-tuberculosis therapy. MTBDRsl assay was performed for molecular detection of mutations. Sequence analysis was performed for characterization and mutational profiling of FQ resistant isolates. Results FQs resistance was observed in 104 (18.5\%) samples and most of them were previously treated and treatment failure cases. A total of 102 isolates had mutations in gyr A gene, D94G and $\mathrm{A} 90 \mathrm{~V}$ being the most prevalent while gyr $B$ gene mutations were observed in only two isolates. Some substitutions in amino acid $\mathrm{D}, \mathrm{A}$ and $\mathrm{T}$ were also determined by sequence analysis in hybridization pattern of both co-existence cases which appeared with both wild type and mutant probe and with missing wild type probe as well. Conclusion The findings suggest that the genotypic studies for FQs resistance should be carried out at time of initial diagnosis, before starting treatment, to rule out all type of mutations for potential use in the treatment and to control its resistance.

\section{Background}

Pakistan is among the thirty high Tuberculosis (TB) burden countries where complete elimination of TB is, unfortunately, a distant reality. TB is ranked in ninth number with leading cause of mortality worldwide where, about one third of the world's population is infested with latent infection with TB [1]. According to the World health organization (WHO) 2018 annual report, the incidence rate of TB in Pakistan is $267 / 100,000$ population where mortality rate is reported as $27 / 100,000$ (excludes HIV co-infection cases) [2]. There is significant number of population where TB remains undiagnosed and untreated

The phenotypic resistance to flouroquinolones (FQs) is associated with mutation in gyr A and gyr B gene in quinolone resistance-determining region (QRDR), a type II DNA topoisomerase, which target DNA gyrase. Mutations in the DNA gyr subunit A confer the high level resistance whereas other confers low level resistance to gyr subunit B [3]. FQs have long been using as anti-tuberculosis drugs and their wide spread use has led to the development of resistance in clinical isolates of Mycobacterium tuberculosis (Mtb). Recently, it is reported that this high degree of resistance to FQs in multidrug-resistant (MDR) cases is a major risk factor as it can lead to the development of XDR-TB $[4,5]$.

Geographic differences exist in the frequency of gyr A mutations. The understanding of frequency and geographic distribution of the FQs mutation is important in order to maximize its sensitivity and specificity. Mutations in codon 88-94 of QRDR of gyrA gene appear most commonly particularly in codons $88,90,91$, and 94 . The mutations in the region of codon 500 and 538 of $\mathrm{gyr} B$ gene are most often associated with FQs resistance [6].

The emergence of drug resistance and persistence of infection is serious threat to control TB [7]. This high incidence of resistance severely limits treatment options and requires the use of more toxic and 
costly treatment regimens [8]. The present study is conducted to detect the mutational profile of FQs-R to help in determination of their potential utility and selection of adequate drug regimens.

\section{Methods}

\section{Sample collection}

Mycobacterium tuberculosis isolates were procured from patients diagnosed with pulmonary TB and had been on anti-tuberculosis therapy. All type of patients with newly diagnosed cases, category I failure, retreatment and relapse cases were included in the study. Sputum samples were collected from the patients and this study was approved by the Research Ethics and Biosafety Committee (No.D/650/MMG) of Department of Microbiology and Molecular Genetics, University of the Punjab, Lahore, Pakistan.

\section{Sample processing}

A total of 562 samples were used for antimicrobial testing. Initially, the sputum samples were decontaminated using standard NALC-NaOH (N-acetyl-L-cysteine sodium hydroxide) method [9] and smear positive samples were directly processed for DNA extraction. Smear negative samples were primarily cultured on MGIT Bactec $960 \AA$ medium. DNA was extracted using Genolyse version 1.0 kit method (Hain Lifescience, Germany). After DNA extraction, supernatant was collected and transferred into fresh tube and stored at $-20^{\circ} \mathrm{C}$ for further processing.

\section{Molecular detection of FQs resistance}

The phenotypic resistance to second line drugs including flouroquinolones using GenoType MTBDRs/ version 2.0 kit method. The whole procedure of molecular detection with GenoType MTBDRs/ includes three steps i.e. DNA extraction, amplification with biotinylated primers and reverse hybridization. The test was considered valid when all control bands appeared correctly.

\section{PCR and sequencing}

Primers were designed against QRDR region of gyrA gene forward primer 5-

GATGCAGCGCAGCTACATCGAC-3 and Reverse primer 5-GATGCAGCGCAGCTACATCGAC-3. The cyclic parameters for the amplification reaction were $95^{\circ} \mathrm{C}$ for $5 \mathrm{~min}$ followed by 40 cycles of $95^{\circ} \mathrm{C}$ for $30 \mathrm{sec}$, $61^{\circ} \mathrm{C}$ for $45 \mathrm{sec}, 72^{\circ} \mathrm{C}$ for $50 \mathrm{sec}$ and final elongation for $10 \mathrm{~min}$ at $72^{\circ} \mathrm{C}$. The PCR products were purified using a Qiagen PCR purification kit and eluted in TE buffer. The sequencing of the isolates was performed by $\left.\right|^{\text {st }}$ Base sequencer, Malaysia. 


\section{Results}

\section{Detection of FQs resistance}

The samples were collected from seven different districts of Punjab, Pakistan. The frequency of FQS-R and its mutational profiling was determined in these regions. Genotype MTBDRsl assay was performed to determine resistance to second line drugs. Both $g y r A$ and $g y r B$ were examined for FQs resistance. A total of $104 / 562(18.5 \%)$ samples were found resistant to FQs.

Among FQs resistant isolates, 8 isolates were rifampcin sensitive, 8 were mono resistant (resistant for rifampcin and sensitive for isoniazid) and 88 were MDR (multi drug resistant). Most of the patients were previously treated and appeared with category 1 failure treatment and some were on category 4 treatment. In total, 102/104 showed the resistance for gyrA gene while gyrB gene mutations were prevalent only in 2/104 cases with mutation in E540V. The resistance was interpreted according to the presence and absence of wild type and mutant probe. When all wild type probes appeared it was interpreted with no detectable mutation. In the absence of any wild type probe, the respective amplicon cannot bind to the corresponding wild type probe resulting in detectable mutation.

\section{Mutational profiling of gyrA gene}

The mutation probes detect some of the most common resistance-mediating mutations. Table 1 shows all types of mutations in the gyrA gene and pattern of corresponding wild and mutant bands. Failure of the gyrA WT3 band and the development of MUT3C was the most common pattern of mutation. Mutational analysis showed that these mutations mostly alter the protein at codon 94 and 90 which represents the replacement of aspartic acid with glycine and alanine with valine, respectively. Aspartic acid has also replaced with alanine, asparagine and tyrosine in other types of mutations at codon 94 . Mutation at codon 91 was also found where serine was replaced with proline.

Different hybridization patterns were observed for FQ resistance in gyrA gene, 19 isolates from all possible patterns for further mutational analysis in the hot spot regions of gyrA gene loci using H37RV (AGH06049.1) as a reference strain. In these isolates amino acid changes were determined due to these mutations. We found variation in four isolates with respect to wild type and mutant probe where amino acid change vary from the most known mutation reported by LPA testing. One isolate substituted from asp $\rightarrow$ ala, two isolates had ala $\rightarrow$ val and one had thr $\rightarrow$ ala in additionally with ser/ala $\rightarrow X$ (undetrmined) (Fig. 1).

\section{Discussion}

FQs have long been widely used for several infectious diseases and easily accessible in our region even without prescription. Such misuse of FQs has highly contributed to their efficacy in the treatment of TB 
and emergence of FQ-resistance. MTB develop resistance against FQs, mostly, by developing mutations against drug targeted proteins. In the present study, these hot spot mutations were characterized by sequence analysis in QRDR of gyrA gene prevalent in the target region. The study group involved the defaulted, failure or retreatment cases and these patients treatment regimen include FQs. Initially, we found resistance against second line drugs by Genotype MTBDRsl assay. The results of all isolates were interpreted according to the guidelines. A total of 104/562 isolates were found resistant to FQs, of them only two isolates had shown resistance with mutation in E540V. The high level of FQs resistance was also reported in other provinces of Pakistan [10,11] and neighboring countries India [12,13], China [14,15] and Bangladesh [16,17].

It was found that most of the isolates had mutation in gyr A gene loci with substitution at amino acid 94 and 90 where $D 94 G$ and $A 90 \mathrm{~V}$ were most often. These mutations are associated with high level resistance to FQs. D94N/Y, D94A and S91P were other mutations found in our isolates. Our findings correlate with most of the previous studies $[6,18,19]$.

Since Genotype MTBDRsl assay target only small region of gene with limited number of well-known mutations and sometimes, the interpretations are indistinct for cross-resistance to FQs which occur due to particular gyrA mutations. So, the sequence analysis was performed for understanding of resistance on the genotypic level [20]. In all sequenced isolates (except three), S95T mutation was found with other important mutations. This type of mutation is found even in sensitive isolates and not associated with FQs resistance as determined by other studies $[21,22]$. The results of all of our mutations are also in line with a study in Pakistan where they detect the mutations in extensively drug resistant strains [23].

Interestingly, we had found some other hot spot mutations in hybridization pattern of co-existence cases where no wild type probe was missing and a mutant probe was also present. In our two isolates, when MUT3C and MUT2 appeared in the presence of its all wild type probe, substitution of Ala into Val was observed, but when MUT2 probe appeared in the absence of WT3 the mutational change of Asp into Ala occurred. Four isolates selected for our most common pattern i.e. absence WT3 and presence of corresponding mutant probe MUT3C, but, one isolate had shown mutation of Thr into Ala additionally with $\mathrm{G} / \mathrm{M} / \mathrm{R}$ into undetermined amino acid $\mathrm{X}$. For most of the isolates amino acids changed from the pattern of well-known mutations but mostly occurred in the same region i.e. codon 94 and 91 . Overall, these findings suggest that the mutation pattern can differ according to the hybridization pattern of wild type and a mutant probe.

\section{Conclusions}

In conclusion, the emergence of flouroquinlone resistance in clinical isolates is alarming. We found high proportion of FQs resistance in MDR cases and even in mono-resistant and all drugs sensitive isolates. Previously treated and the failure TB treatment cases being the most important group for developing resistance. Our findings suggest that the implementation of FQs in these patients should be carefully 
administered and genotypic studies should be carried out, preferably at the time of initial diagnosis, to rule out all type of mutations for effective treatment and particularly to control its resistance.

\section{Abbreviations}

FLQ: Flouroquinolone; FLQ-R: Flouroquinolone Resistant; LPA: Line Probe Assay; MDR: Multidrug Resistant; MGIT: Mycobacteria Growth Indicator Tube; Mtb: Mycobacterium tuberculosis; MUT: Mutant; NALC-NaOH: N-acetyl-L-Cysteine Sodium Hydroxide; QRDR: Quinolone Resistance Determining Region; TB: Tuberculosis; WT: Wild Type; XDR: Extensively Drug Resistant.

\section{Declarations}

\section{Ethical approval and consent to participate}

All study participants were informed verbally (approved by Ethics and Biosafety Committee) and consent to participate was collected from the patients directly or from parents. No study participants were included in the present study involving guardians. The study was approved by Departmental Research Ethics and Biosafety Committee.

\section{Consent for publication}

"Not Applicable".

\section{Availability of data and materials}

The datasets generated and/ or analyzed during the current study are part of PhD thesis of the first author and not publicly available. The datasets are available from the corresponding author on reasonable request.

\section{Competing interests}

The authors declare that they have no competing interests.

\section{Funding}

No funding was obtained for the current study.

\section{Authors' contributions}

SK performed experiments, analyzed the data and wrote the manuscript.conceived the research idea. ZT provided sources. NM helped in experiments and data analysis. Both MS had responsibility for data collection. AR conceived the research idea and helped in manuscript preparation. All authors contributed substantially to the interpretation of the results. All authors approved the final manuscript. 
Acknowledgements

"Not Applicable".

\section{References}

[1] Muñoz L, Stagg HR, Abubakar I Diagnosis and management of latent tuberculosis infection. Cold Spring Harb Perspect Med. 2015;5(11): a017830.

[2] WHO Global tuberculosis report 2018. Available from URL https://www.who.int/tb/publications/global_report/gtbr2018_annex3.pdf?ua=1

[3] Zhang Y, Yew WW. Mechanisms of drug resistance in Mycobacterium tuberculosis [State of the art series. Drug-resistant tuberculosis. Edited by CY. Chiang. Number 1 in the series]. Int J Tuberc Lung Dis. 2009;13(11):1320-1330.

[4] Castro RA, Ross A, Kamwela L, Reinhard M, Loiseau C, Feldmann J, Borrell S, Trauner A, Gagneux S. The evolution of fluoroquinolone-resistance in Mycobacterium tuberculosis is modulated by the genetic background. bioRXiv. 2019;2019: 659045.

[5] Sharma R, Sharma SK, Singh BK, Mittal A, Kumar P. High degree of fluoroquinolone resistance among pulmonary tuberculosis patients in New Delhi, India. Indian J Med Res. 2019;149(1): 62-66.

[6] Avalos E, Catanzaro D, Catanzaro A, Ganiats T, Brodine S, Alcaraz J, Rodwell T. Frequency and geographic distribution of $g y r A$ and $g y r B$ mutations associated with fluoroquinolone resistance in clinical Mycobacterium tuberculosis isolates: a systematic review. PLoS One 2015;10(3): e0120470.

[7] Kaur D, Kutum R, Dash D, Brahmachari SK. Data Intensive genome level analysis for identifying novel, non-toxic drug targets for multidrug resistant Mycobacterium tuberculosis. Sci Rep. 2017;7: 46595.

[8] Shah NS, Auld SC, Brust JC, Mathema B, Ismail N, Moodley P, Mlisana K, Allana S, Campbell A, Mthiyane T, Morris N, Mpangase P, van der Meulen H, Omar SV, Brown TS, Narechania A, Shaskina E, Kapwata T, Kreiswirth B, Gandhi NR. Transmission of extensively drug-resistant tuberculosis in South Africa. N Engl J Med. 2017;376(3): 243-253.

[9] Stinson KW, Eisenach K, Kayes S, Matsumoto M, Siddiqi S, Nakashima S. et al. Mycobacteriology Laboratory Manual, global laboratory initiative advancing TB diagnosis. $1^{\text {st }} e d$. April 2014.

[10] Jabeen K, Shakoor S, Malik F, Hasan R (2015) Fluoroquinolone resistance in Mycobacterium tuberculosis isolates from Pakistan 2010-2014: Implications for disease control. Int J Mycobacteriol. 2015;4:47-48.

[11] Akhtar AM, Arif MA, Kanwal S, Majeed S. Prevalence and drug resistance pattern of MDR TB in retreatment cases of Punjab, Pakistan. J Pak Med Assoc. 2016;66(8): 989-3. 
[12] Desai U, Joshi JM. Extrapulmonary drug-resistant tuberculosis at a drug-resistant tuberculosis center, Mumbai: Our experience-Hope in the midst of despair! Lung India 2019;36(1): 3-7.

[13] Sharma AK, Gupta N, Kala DK, Patni T, Dixit R, Verma S, Chandran A. A study on pattern of resistance to second line anti tubercular drugs among multi drug resistant tuberculosis patients. Indian $\mathrm{J}$ Tuberc. 2018;65(3): 233-236.

[14] Gao Y, Zhang Z, Deng J, Mansjö M, Ning Z, Li Y, Xu B. Multi-center evaluation of GenoType MTBDRsl line probe assay for rapid detection of pre-XDR and XDR Mycobacterium tuberculosis in China. $J$ Infect. 2018;77(4): 328-334.

[15] Wang Z, Xie T, Mu C, Sun R, Wang C, Zhao H, Ju H. Performance of sequencing in predicting ofloxacin resistance in Mycobacterium tuberculosis from positive Bactec MGIT 960 cultures. Ann Clin Lab Sci. 2018;48(1): 69-74.

[16] Rigouts L, Coeck N, Gumusboga M, de Rijk WB, Aung KJM, Hossain MA, Van Deun A. Specific gyrA gene mutations predict poor treatment outcome in MDR-TB. J Antimicrob Chemother. 2015;71(2): 314323.

[17] Kamal SMM, Hossain A, Sultana S, Begum V, Haque N, Ahmed J, Chowdhury RA. Anti-tuberculosis drug resistance in Bangladesh: reflections from the first nationwide survey. Int. J Tuberc Lung Dis. 2015;19(2): 151-156.

[18] Von Groll A, Martin A, Jureen P, Hoffner S, Vandamme P, Portaels F, Palomino JC, da Silva PA. Fluoroquinolone resistance in Mycobacterium tuberculosis and mutations in gyrA and gyrB. Antimicrob Agents Chemother. 2009;53(10): 4498-4500.

[19] Singhal R, Reynolds PR, Marola JL, Epperson LE, Arora J, Sarin R, Myneedu VP, Strong M, Salfinger $M$. Sequence analysis of fluoroquinolone resistance-associated genes gyrA and gyrB in clinical Mycobacterium tuberculosis isolates from patients suspected of having multidrug-resistant tuberculosis in New Delhi, India. J Clin Microbiol. 2016;54(9): 2298-2305.

[20] Schön T, Miotto P, Köser CU, Viveiros M, Böttger E, Cambau E. Mycobacterium tuberculosis drugresistance testing: challenges, recent developments and perspectives. Clin Microbiol Infect.

2017;23(3):154-160.

[21] Giannoni F, Iona E, Sementilli F, Brunori L, Pardini M., Migliori B, Orefici G, Fattorini L. Evaluation of a new line probe assay for rapid identification of gyrA mutations in Mycobacterium tuberculosis. Antimicrob Agents Chemother. 2005;49(7):2928-2933.

[22] Juarez-Eusebio DM, Munro-Rojas D, Muñiz-Salazar R, Laniado-Laborín R, Martinez-Guarneros JA, Flores-López CA, Zenteno-Cuevas R. Molecular characterization of multidrug-resistant Mycobacterium 
tuberculosis isolates from high prevalence tuberculosis states in Mexico. Infect Genet Evol. 2017;55: 384391.

[23] Ali A, Hasan R, Jabeen K, Jabee N, Qadeer E, Hasan Z. Characterization of mutations conferring extensive drug resistance to Mycobacterium tuberculosis isolates in Pakistan. Antimicrob Agents chemother. 2011;55(12):5654-5659.

\section{Table}

Table 1: The frequency and the mutations conferring resistance to FQs.

\begin{tabular}{llllc}
\hline $\begin{array}{l}\text { gyrA Mutation } \\
\text { probe }\end{array}$ & $\begin{array}{l}\text { Missing wild type } \\
\text { probe }\end{array}$ & $\begin{array}{l}\text { Phenotypic } \\
\text { Susceptibility }\end{array}$ & Mutation & $\begin{array}{l}\text { Frequency } \\
(\mathrm{n}=102)\end{array}$ \\
\hline gyr A MUT1 & gyr A WT2 & Resistant & A90V & 25 \\
\hline gyr A MUT2 & gyr A WT2 & Resistant & S91P & 7 \\
& gyr A WT3 & & & 7 \\
\hline gyr A MUT3A & gyr A WT3 & Resistant & D94A & 9 \\
\hline gyr A MUT3B & gyr A WT3 & Resistant & D94N/Y & 45 \\
\hline gyr A MUT3C & gyr A WT3 & Resistant & D94G & 9 \\
\hline None & $\begin{array}{l}\text { any one wild type } \\
\text { probe }\end{array}$ & Resistant & A90V D94A \\
& & D94G & \\
\hline
\end{tabular}

\section{Figures}

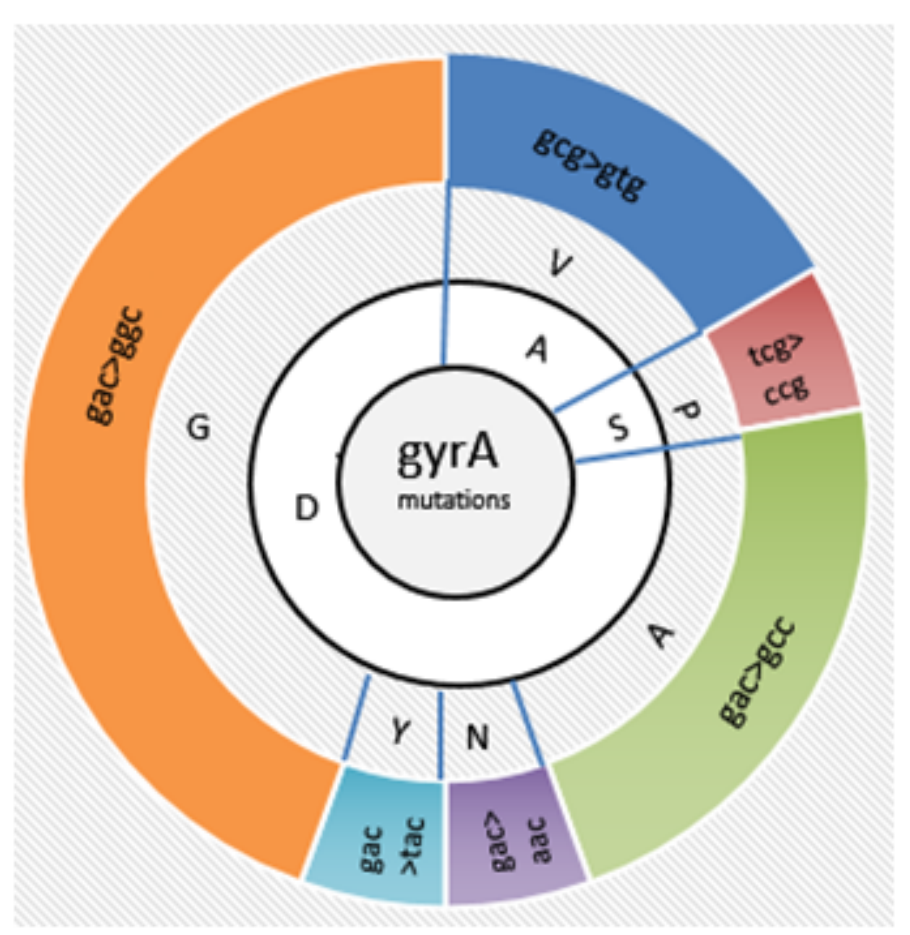

Figure 1 
Frequency amino acid and nucleotide change in FQs isolates. In general, amino acid change had found in three amino acids alanine ( $A$ into $V$ ), serine ( $S$ into $P$ ) and aspartic acid ( $D$ either into $A, N, Y$ or $G$ ). The nucleoide change corresponding to these amino acids change is also represented. 